\title{
Evaluation of Hypervascular Focal Liver Lesions Utilizing Virtual Monoenergetic Images from Third-Generation Dual-Source Dual-Energy Computed Tomography
}

\author{
Niyas Narappulan ${ }^{1}$ Venkatesh Kasi Arunachalam ${ }^{1} \quad$ Ezhilmathi Alavandar $^{1} \quad$ Swathigha Selvaraja $^{1}$ \\ Rupa Renganathan ${ }^{1}$ Mathew Cherian ${ }^{1}$ \\ ${ }^{1}$ Department of Radiology, Kovai Medical Center and Hospital, \\ Coimbatore, India \\ J Gastrointestinal Abdominal Radiol ISGAR 2022;5:85-93.
}

\author{
Abstract \\ Keywords \\ - third generation \\ - dual source \\ - dual energy \\ - virtual \\ monoenergetic \\ image \\ - hypervascular \\ - liver
}

Objectives The purpose of our study was to evaluate the virtual monochromatic imaging in detecting hypervascular focal liver lesions in the late arterial phase with third-generation dual-source dual-energy computed tomography and to assess its image quality.

Materials and Methods In our study, 80 patients were included. Contrast-enhanced images in the late arterial phase (in the dual-energy mode) were acquired and were post-processed in Syngo, via workstation, using Monoenergetic + software. Five sets of images, one polychromatic energy image (corresponding to $120 \mathrm{kVp}$ single-energy image) and four virtual monoenergetic image (VMI) sets at 40, 50, 60, and $70 \mathrm{keV}$ levels, were generated. All these images were analyzed both objectively and subjectively. The attenuation values were measured, and the contrast-to-noise ratio (CNR) of liver and tumor were measured and compared objectively in each dataset. Image noise, image contrast, and diagnostic confidence for liver lesion detection were analyzed subjectively using a five-point scale system. Statistical analysis was performed using Kolmogorov-Smirnov, analysis of variance, and Kruskal-Wallis tests.

Results Among the VMI, maximum image noise was observed in the $40 \mathrm{keV}$ image, with a gradual reduction in the image noise being noted with an increase in the VMI energy. The CNR of the hepatic parenchyma and the tumor gradually increased with a reduction in VMI energy from 70 to $40 \mathrm{keV}$. On subjective analysis, image contrast and image noise were observed to be more in low VMI datasets. In lesion detection, diagnostic confidence with an excellent confidence level was observed with a decrease in VMI energy.

Conclusion VMI datasets of 40 to $70 \mathrm{keV}$ from third-generation dual-source DECT provide superior diagnostic accuracy for detecting hypervascular liver lesions. Considering the image noise and lesion detection rate among the VMI datasets, $60 \mathrm{keV} \mathrm{VMI} \mathrm{is}$ the most helpful dataset for increased liver lesion detection with good image quality. published online

February 17, 2022
DOI https://doi.org/

$10.1055 / \mathrm{s}-0042-1742677$. ISSN 2581-9933. (c) 2022. Indian Society of Gastrointestinal and Abdominal Radiology. All rights reserved.

This is an open access article published by Thieme under the terms of the Creative Commons Attribution-NonDerivative-NonCommercial-License, permitting copying and reproduction so long as the original work is given appropriate credit. Contents may not be used for commercial purposes, or adapted, remixed, transformed or built upon. (https://creativecommons.org/ licenses/by-nc-nd/4.0/)

Thieme Medical and Scientific Publishers Pvt. Ltd., A-12, 2nd Floor, Sector 2, Noida-201301 UP, India 


\section{Introduction}

The recent revolution in computed tomography (CT) technology is the introduction of dual-energy technology. Dualenergy CT (DECT) improves lesion detection and characterization beyond the current level achievable with conventional CT. There are several advanced applications possible with DECT when imaging the liver, and a few of these include detection and staging of hypervascular liver lesions, determining the components of a hepatic lesion, which helps to characterize the lesions, and differentiating simple cysts from hypovascular metastasis using water and iodine density images. ${ }^{1,2}$

One of the post-processed images from the dual-energy data is the virtual monoenergetic image (VMI). Theoretically, a series of VMI of various kilo-electron-volt ( $\mathrm{keV}$ ) levels could be calculated by scanning at two different energy levels. When a polychromatic X-ray beam is transmitted through the patient's body, the low-energy photons are absorbed and increase the mean energy of the X-ray beam. When the mean energy of the beam increases, it moves away from the K-edge of the iodine (33.2 keV). This results in decreased attenuation of the X-rays by iodine-containing tissues, which leads to a decrease in contrast resolution between the objects. ${ }^{3}$ VMI from DECT represent how the tissues would attenuate if exposed to the monoenergetic X-ray beam. In a low keV VMI, since the energy of the X-ray beam is close to the K-edge of iodine, there will be more photoelectric absorption of X-ray photons by iodinecontaining structures. This increases the Hounsfield unit (HU) of iodine-containing tissues and increases the contrast resolution between the objects. Previous studies have demonstrated that spectral CT provides a set of monochromatic images for increased detectability of small hepatocellular carcinoma (HCC) at energy levels of 40 to $70 \mathrm{keV}$ by improving contrast-to-noise ratio (CNR) values during the late arterial phase with no or only minimal dose penalty compared with singleenergy CT (SECT) acquisition. ${ }^{4-6}$

Our study was aimed to study monochromatic imaging in detecting hypervascular focal liver lesions in the late arterial phase with third-generation dual-source dual-energy CT (DSCT) and to assess its image quality.

\section{Materials and Methods}

The local ethical and scientific committee had approved this study, and written informed consent was obtained from all participants. The study period was from July 2019 to August 2020. The exclusion criteria included general contraindications for iodinated contrast and incomplete study due to various reasons like anaphylactic reaction, uncooperative patient, low image quality, and extensive artifacts. A total of 80 lesions were taken for the study from 80 patients after exclusion.

\section{Methodology}

\section{Examination Protocol}

All scans were performed on the third-generation DSCT (Somatom Force, Siemens). Automated tube-current modu- lation (CARE Dose4D, Siemens) was activated in all examinations. Contrast-enhanced images in the late arterial phase (in the dual-energy mode), portal venous, and delayed phases (the single-energy mode) were acquired.

The dual-energy mode was acquired using the following parameters: pitch: 0.6 , field of view: 512 , tube $A: 100 \mathrm{kVp}$, and tube $\mathrm{B}: 150 \mathrm{kVp}$. The single-energy mode was acquired using the following parameters: pitch: 0.6 , field of view: 512 , and tube voltage: $120 \mathrm{kV}$.

Iodinated intravenous contrast was injected at a flow rate of $4 \mathrm{~mL} / \mathrm{s}$ through an 18 gauge IV access placed in the right median cubital vein. The volume of contrast injection was calculated based on the patient's weight $(1 \mathrm{~mL} / \mathrm{kg})$. The scan was timed using a bolus tracking software application. The scan was triggered with a delay of 15 to 20 seconds (after bolus tracking) for the late arterial phase, 50 to 60 seconds for the portal phase, and another delay of 2 minutes for the delayed phase. The scan covered from the dome of the diaphragm to the iliac crest level in arterial and delayed phases and from the diaphragm to the lower end of the ischial tuberosity in the venous phase.

After completing image acquisition, images were transferred and post-processed in Syngo, via (version VA), using Monoenergetic + , Siemens healthcare software. VMI for this study was generated from the dual-energy images acquired during the late arterial phase. These images were postprocessed to derive five sets of images, one polychromatic energy image (PEI) (corresponding to $120 \mathrm{kVp}$ single-energy image) and four VMI sets at 40, 50, 60, and $70 \mathrm{keV}$ levels. All axial images were reconstructed in $1 \mathrm{~mm}$.

\section{Image Analysis}

The images were analyzed both subjectively and objectively.

\section{Objective Image Analysis}

Objective image analysis was done by comparing the $\mathrm{HU}$ values. The attenuation of erector spinae muscle, liver tumor, and adjacent liver parenchyma was measured by placing the circular region of interest (ROI). When there were many lesions in one patient, the largest lesion (in terms of maximal diameter) was selected for better objective lesion analysis. Each ROI (minimum ROI area was $1 \mathrm{~cm}^{2}$ ) placement and measurement was done thrice to ensure consistency, and the averaged value was considered for analysis. The image noise was defined as the standard deviation (SD) of the HU muscle. The CNR of the liver can be calculated as follows:

The CNR of the liver $=\mathrm{HU}$ liver $-\mathrm{HU}$ muscle/image noise.

The lesion CNR can be calculated by dividing the lesion-toliver contrast by the image noise.

The CNR of the lesion $=$ HU tumor $-\mathrm{HU}$ liver/image noise.

\section{Subjective Analysis}

The subjective qualities of PEI and VMI at 40 to $70 \mathrm{keV}$ energies were evaluated using the five-point scale for image contrast, image noise, and diagnostic confidence for liver lesion detection. Patient information and acquisition parameters were blinded to reduce observer bias. The images were randomized and presented using a soft-tissue window, with 
window adjustment being encouraged for appropriate image interpretation.

Five-point scales were used, and the scores were as follows:

Image contrast $=1$-undiagnostic, 2-suboptimal, 3-average, 4-good, and 5-excellent.

Image noise $=1$-undiagnostic, 2-severe, 3-moderate, 4 -mild, and 5-absent.

Diagnostic confidence for liver lesion detection $=1-$ undiagnostic; 2-suboptimal, low confidence; 3-average; 4-above average; and 5-most confident.

\section{Statistical Analysis}

Statistical Package for Social Sciences version 20 (IBM, United States) was employed to analyze data. The results on continuous measurements were presented on mean $\pm S D$, and categorical measurements were presented in number/percentage. For continuous variables, the Kolmogorov-Smirnov test was used to assess the normality of the data. Analysis of variance and Kruskal-Wallis test (nonnormal distribution) were used to assess the significant difference between more than two independent group means. Fisher's exact test was used for testing the association between two categorical variables. A p-value of 0.05 was considered as the level of significance.

\section{Results}

\section{Study Population}

A total of 80 patients were included. The sample group's mean age was 59.6 years, ranging between 21 and 81 years. Among 80 patients, males accounted for $72.5 \%$ of the total and females were $27.5 \%$.

\section{Diagnosis}

Patients were presented with varied clinical profiles and diagnoses, among which HCC and metastasis were the most common proven/suspected diagnosis in our study. Twentynine patients were diagnosed with HCC, 25 of them with features of cirrhosis and four without features of cirrhosis. In 27 patients with known malignancy, hypervascular liver lesions detected were considered metastasis. Lesions in 15 patients were diagnosed as hemangioma. Others in the diagnosis category include nine cases: one case of hepatic adenoma, one case of focal nodular hyperplasia, and seven cases of hypervascular dysplastic nodules (Liver Imaging Reporting and Data System [LIRADS] 3 lesions). Among these 80 patients, histopathology confirmation was available for 23 patients (seven cases of HCC and 16 cases of metastasis).

\section{Objective Analysis}

\section{Image Noise}

The mean image noise of the PEI was $12.5 \pm 2.6 \mathrm{HU}$. Among the VMI, maximum image noise was observed in the $40 \mathrm{keV}$ image, $35.0 \pm 6.0 \mathrm{HU}$ (-Table 1). A gradual reduction in the image noise was noted with an increase in the VMI energy.
Table 1 Demonstrating image noise at different energies

\begin{tabular}{|l|l|}
\hline & Image noise \\
\hline $\mathrm{PEI}$ & $12.5 \pm 2.6$ \\
\hline $40 \mathrm{keV}$ & $35.0 \pm 6.0$ \\
\hline $50 \mathrm{keV}$ & $25.6 \pm 4.4$ \\
\hline $60 \mathrm{keV}$ & $19.7 \pm 3.7$ \\
\hline $70 \mathrm{keV}$ & $16.3 \pm 3.3$ \\
\hline
\end{tabular}

Abbreviation: PEl, polychromatic energy image.

\section{Contrast-to-Noise Ratio of Hepatic Parenchyma and Tumors}

The CNR of the hepatic parenchyma and tumors (80 lesions from 80 patients) gradually increased as the monoenergetic level decreased.

\section{Contrast-to-Noise Ratio of the Hepatic Parenchyma}

The CNR of hepatic parenchyma in VMI was significantly higher at $40 \mathrm{keV}$ and lowest at $70 \mathrm{keV}$ (hepatic CNR ranges from $1.27 \pm .86 \mathrm{HU}$ at $40 \mathrm{keV}$ to $0.73 \pm 0.76 \mathrm{HU}$ at $70 \mathrm{keV}$, $p<0.001)$. Although CNR is the lowest for $70 \mathrm{keV}$ images, it was statistically significant from the PEI $(0.62 \pm 0.48)$ (-Fig. 1). The sample size used to calculate the CNR of the liver was 63. A total of 17 cases were excluded while calculating the CNR of liver because of severe fatty liver in these patients, decreasing the $\mathrm{HU}$ of the liver sometimes to a negative value, which may lead to false-negative results.

\section{Contrast-to-Noise Ratio of Tumor}

Like the CNR of the hepatic parenchyma, the CNR of the tumor also gradually increased with a reduction in VMI energy from 70 to $40 \mathrm{keV}$ (the tumor CNR at $40 \mathrm{keV}$ was $2.5 \pm 1.70 \mathrm{HU}$ and at $70 \mathrm{keV}$ was $1.44 \pm 1.08 \mathrm{HU})$. The CNR of the studied VMI (40 to $70 \mathrm{keV}$ ) was considerably more than the CNR of PEI of $1.03 \pm 1.39(p<0.001)$ ( - Table 2 )

\section{Subjective Analysis}

\section{Image Contrast}

On subjective analysis, the image contrast of VMI at 40 to $70 \mathrm{keV}$ was rated as superior to PEI ( $\mathrm{p}<0.001)$. In total, $33.8 \%$ of lesions were labeled as excellent image contrast in the

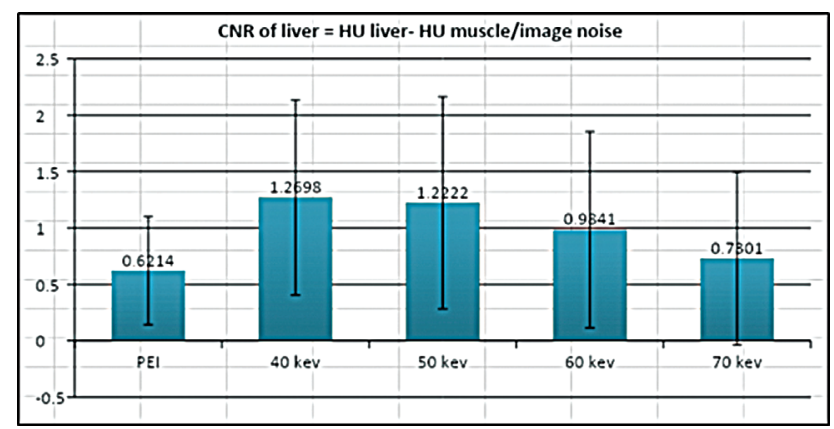

Fig. 1 CNR of the liver at various energy levels. CNR, contrast-to-noise ratio. 
88 Virtual Monoenergetic Imaging in Third Generation Dual source Dual Energy CT Narappulan et al.

Table 2 Comparison of CNR tumor at different energy levels

\begin{tabular}{|l|l|}
\hline & CNR tumor \\
\hline PEI & $1.031 \pm 1.3967$ \\
\hline $40 \mathrm{keV}$ & $2.525 \pm 1.7083$ \\
\hline $50 \mathrm{keV}$ & $2.171 \pm 1.4849$ \\
\hline $60 \mathrm{keV}$ & $1.824 \pm 1.2906$ \\
\hline $70 \mathrm{keV}$ & $1.439 \pm 1.0859$ \\
\hline
\end{tabular}

Abbreviations: CNR, contrast-to-noise ratio; PEI, polychromatic energy image.

$40 \mathrm{keV}$ images. Similarly, $15 \%$ of the lesions in $50 \mathrm{keV}, 10 \%$ of the lesions in $60 \mathrm{keV}$, and $3.8 \%$ of the lesions in $70 \mathrm{keV}$ were labeled as excellent contrast images (- Table 3 ). In comparison, no lesions were marked as having excellent image contrast in PEI.

\section{Image Noise}

The image noise was considerably higher in the VMI at 40 to $60 \mathrm{keV}$ such that the average image noise was observed in $65 \%$ of the cases in $40 \mathrm{keV}$ image, $67.5 \%$ cases in $50 \mathrm{keV}, 57.5 \%$ cases in $60 \mathrm{keV}$, and 45\% cases in the $70 \mathrm{keV}$ (-Table 4). However, average image noise was observed only in $2.5 \%$ of the cases in the PEI. Thus, 65\% of the cases had good images and $32.5 \%$ had excellent images with significantly reduced image noise $(p<0.01)$ in PEI.

\section{Diagnostic Confidence for Liver Lesion Detection}

The highest score for diagnostic confidence was assigned for VMI at $40 \mathrm{keV}$, followed by VMI at 50 to $70 \mathrm{keV}$, all of which were better than PEI $(p<0.01)$. From - Table 5, it is evident that $10 \%$ of the cases that were not detectable in the PEI were detectable in the entire VMI at 40 to $70 \mathrm{keV}$. Among the VMI, $46.3 \%$ of cases were detected with excellent confidence at $40 \mathrm{keV}$ images. A decrease in detecting lesions with an

Table 3 Comparison between PEI and VMI with regard to image contrast

\begin{tabular}{|c|c|c|c|c|c|c|c|c|c|c|}
\hline & \multicolumn{2}{|l|}{ PEI } & \multicolumn{2}{|c|}{$40 \mathrm{keV}$} & \multicolumn{2}{|c|}{$50 \mathrm{keV}$} & \multicolumn{2}{|c|}{$60 \mathrm{keV}$} & \multicolumn{2}{|c|}{$70 \mathrm{keV}$} \\
\hline & $n$ & $\%$ & $n$ & $\%$ & $n$ & $\%$ & $n$ & $\%$ & $n$ & $\%$ \\
\hline Un-diagnostic (1) & 3 & 3.8 & - & - & - & - & - & - & 1 & 1.3 \\
\hline Suboptimal (2) & 29 & 36.3 & 1 & 1.3 & 1 & 1.3 & 2 & 2.5 & 11 & 13.8 \\
\hline Average (3) & 44 & 55.0 & 13 & 16.3 & 19 & 23.8 & 25 & 31.3 & 25 & 31.3 \\
\hline Good (4) & 4 & 5.0 & 39 & 48.8 & 48 & 60.0 & 45 & 56.3 & 40 & 50.0 \\
\hline Excellent (5) & - & - & 27 & 33.8 & 12 & 15.0 & 8 & 10.0 & 3 & 3.8 \\
\hline
\end{tabular}

Abbreviations: PEI, polychromatic energy image; VMI, virtual monoenergetic image.

Table 4 Image noise comparison between different energy levels

\begin{tabular}{|l|l|l|l|l|l|l|l|l|l|l|}
\hline & \multicolumn{3}{|l}{ PEI } & \multicolumn{2}{l|}{$40 \mathrm{keV}$} & \multicolumn{2}{l|}{$50 \mathrm{keV}$} & \multicolumn{2}{l|}{ 60 keV } & \multicolumn{2}{l|}{$70 \mathrm{keV}$} \\
\cline { 2 - 12 } & $\boldsymbol{n}$ & $\%$ & $\boldsymbol{n}$ & $\%$ & $\boldsymbol{n}$ & $\%$ & $\boldsymbol{n}$ & $\%$ & $\boldsymbol{n}$ & $\%$ \\
\hline Un-diagnostic (1) & - & - & - & - & - & - & - & - & - & - \\
\hline Suboptimal (2) & - & - & 20 & 25.0 & 7 & 8.8 & 2 & 2.5 & - & - \\
\hline Average (3) & 2 & 2.5 & 52 & 65.0 & 54 & 67.5 & 46 & 57.5 & 36 & 45.0 \\
\hline Good (4) & 52 & 65.0 & 8 & 10.0 & 19 & 23.8 & 32 & 40.0 & 43 & 53.8 \\
\hline Excellent (5) & 26 & 32.5 & - & - & - & - & - & - & 1 & 1.3 \\
\hline
\end{tabular}

Abbreviation: PEI, polychromatic energy image.

Table 5 Comparison of different energy levels for diagnostic confidence of liver lesion detection at different energy levels

\begin{tabular}{|c|c|c|c|c|c|c|c|c|c|c|}
\hline & \multicolumn{2}{|c|}{ PEI } & \multicolumn{2}{|c|}{$40 \mathrm{keV}$} & \multicolumn{2}{|c|}{$50 \mathrm{keV}$} & \multicolumn{2}{|c|}{$60 \mathrm{keV}$} & \multicolumn{2}{|c|}{$70 \mathrm{keV}$} \\
\hline & $n$ & $\%$ & $n$ & $\%$ & $n$ & $\%$ & $n$ & $\%$ & $n$ & $\%$ \\
\hline Un-diagnostic (1) & 8 & 10.0 & - & - & - & - & - & - & - & - \\
\hline Suboptimal (2) & 21 & 26.3 & - & - & - & - & 3 & 3.8 & 19 & 23.8 \\
\hline Average (3) & 47 & 58.8 & 21 & 26.3 & 22 & 27.5 & 27 & 33.8 & 19 & 23.8 \\
\hline Good (4) & 4 & 5.0 & 22 & 27.5 & 33 & 41.3 & 31 & 38.8 & 38 & 47.5 \\
\hline Excellent (5) & - & - & 37 & 46.3 & 25 & 31.3 & 19 & 23.8 & 4 & 5.0 \\
\hline
\end{tabular}

Abbreviation: PEl, polychromatic energy image. 
excellent confidence level was observed with an increase in VMI energy.

\section{Lesion Size}

In our study, the mean lesion size was $25 \pm 16 \mathrm{~mm}$. There were 19 lesions with a size less than or equal to $1 \mathrm{~cm}$, most of which comprised hemangioma/hypervascular dysplastic nodules (LIRADS 3)/hypervascular metastasis. These lesions were suboptimal and undetectable in most of the subjective analysis criteria in the PEI; however, all these lesions were detected well in entire sets of VMI such that best detected in the 40 to $50 \mathrm{keV}$, followed by $60 \mathrm{keV}$ and then $70 \mathrm{keV}$.

\section{Discussion}

In this study, we investigated VMI generated from a thirdgeneration DSCT based on the image quality and lesion detectability for arterially enhancing focal liver lesions using conventional PEI as a reference standard. Most of the patients in our study had background liver cirrhosis. Thirty-three out of eighty lesions were LIRADS 3, 4, and 5 lesions with associated background cirrhosis. Primary diagnoses other than the lesions in the cirrhotic liver included hypervascular metastasis (27 lesions), hemangioma (15 lesions), and dysplastic nodules (seven lesions).

A statistically significant increase in the image noise with a decrease in VMI energy was observed in our study such that $40 \mathrm{keV}$ images had maximum image noise among the VMI studied ( - Fig. 2). PEI had less image noise than all the VMI. The mean image noise in the $70 \mathrm{keV}$ image was $16.30 \pm 3.399$, which was closer to image noise in PEI. The subjective analysis shows that $25,8.8$, and $2.5 \%$ of the lesions were under the suboptimal category for image noise in 40,50 , and $60 \mathrm{keV}$, respectively. There was no lesion under this category in the $70 \mathrm{keV}$ images, similar to PEI.

Similar to our study, few previous studies also mentioned an increase in noise in VMI. Altenbernd et al ${ }^{1}$ concluded that low-kilovoltage peak $(\mathrm{kVp})$ images of dual-energy datasets were more sensitive in detecting hypervascular liver lesions but at the cost of subjective image quality. Yamada et $\mathrm{al}^{7}$ and Sudarski et $\mathrm{al}^{8}$ in 2012 and 2014, respectively, who studied the tube-based DECT-VMI, revealed that the highest CNR of hypovascular metastases could be attained at around $70 \mathrm{keV}$ and substantially increased noise deteriorated the diagnostic value of lower-keV images. Similar results were also mentioned by Caruso et al ${ }^{9}$ and Husarik et $\mathrm{al}^{10}$ in their studies.

However, a study by Nagayama et $\mathrm{a}^{11}$ using dual-layer dual-energy CT (DLCT) concluded that VMI at 40 to $70 \mathrm{keV}$ provided better subjective and objective image quality to evaluate hypovascular liver metastases. The lesion detectability was improved with the use of VMI at $40 \mathrm{keV}$ compared with conventional PEI. They also had reduced image noise with decreased VMI energy. Sellers et al directly compared the imaging performance between different DECT platforms. ${ }^{12}$ He found that the DLCT provided constant noise levels over the full range of energies, whereas tube-based DECT showed substantially increased noise for lower energy levels. The detector-based DECT solution can explain the noise behavior of DLCT-VMI that is distinctly different from tube-based DECT-VMI. In tube-based DECT systems, dualenergy data are post-processed only in the image domain in dual-source, split-beam, and dual-spin DECT systems and in the projection domain in rapid $\mathrm{kVp}$ switching DECT system. Angular and temporal interpolation is required before post-
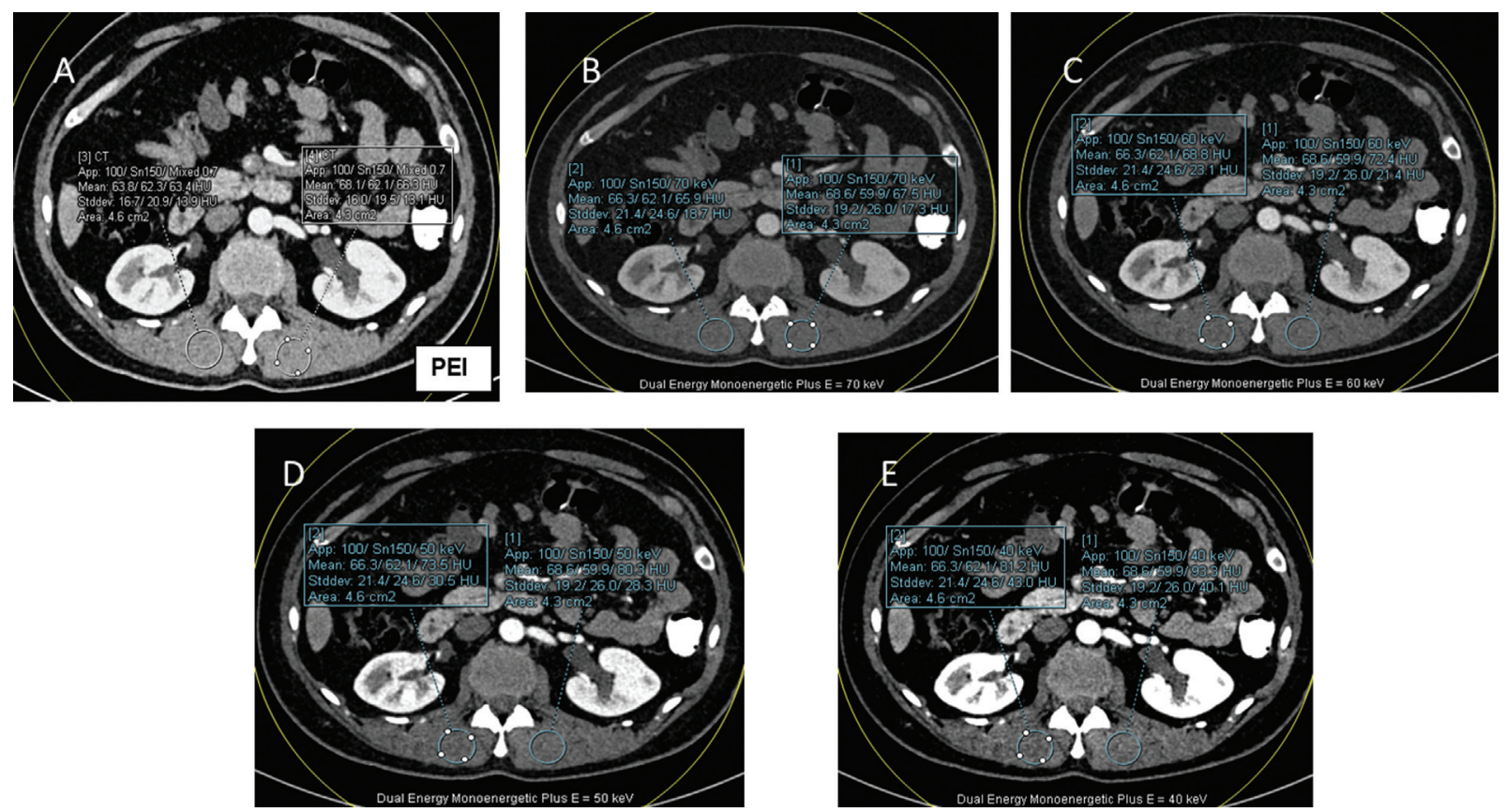

Fig. 2 Axial CT images in the arterial phase (A-PEI and B to E-VMI of 70-40 keV energy) showing measurement of image noise in this study. ROI was placed at erector spinae muscle, and standard deviation was measured at each energy level, which was considered as image noise. Significant increase in image noise from PEI to VMI and further within the VMI from 70 to 40 keV was demonstrated. PEI, polychromatic energy image; ROI, region of interest; VMI, virtual monoenergetic image. 

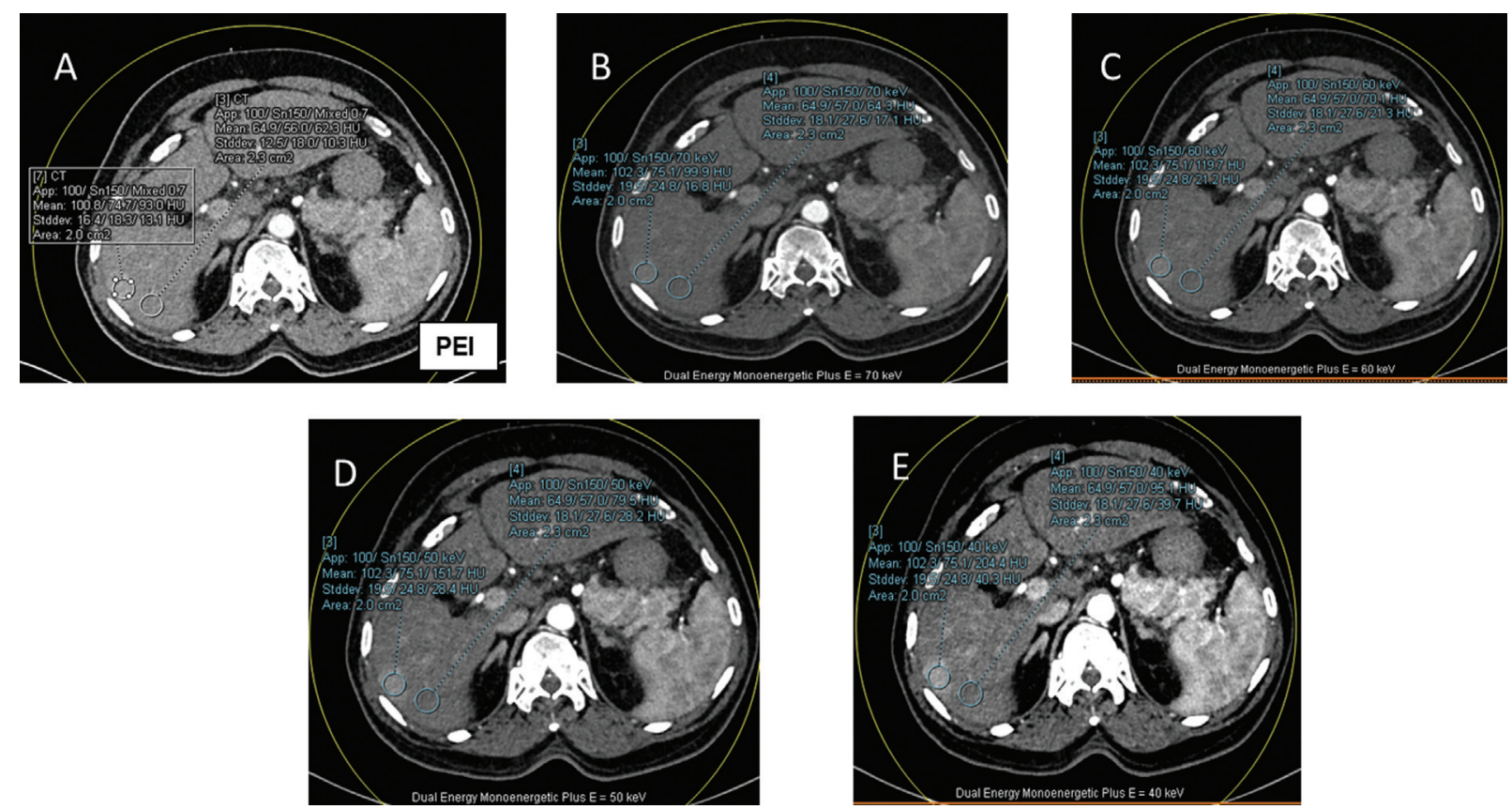

Fig. 3 Axial CT images (A-PEl and B to E-VMI of 70-40 keV energy) of a patient with cirrhotic liver in the arterial phase show a focal arterially hyperenhancing lesion, proven as HCC in segment VI of the right lobe of the liver. This set of images shows measurements of HU tumor and HU liver parenchyma. There was considerable increase in the mean difference between the attenuation of tumor and liver parenchyma from PEI to VMI, and maximum difference was seen in $40 \mathrm{keV}$ image. This makes the lesions better detectable in the VMI at $40 \mathrm{keV}$. HCC, hepatocellular carcinoma; HU, Hounsfield unit; PEI, polychromatic energy image; VMI, virtual monoenergetic image.

processing in the projection domain. This theoretically leads to spatial and temporal resolution deterioration and amplifies the so-called anti-correlated noise, especially at low energies. ${ }^{11}$ The recent advances in dual-source DECT addressed increased noise by introducing an advanced noise-optimized reconstruction algorithm, which provided increased CNR and diagnostic usability with lower-keV VMI for the assessment of hepatic lesions. ${ }^{9,10,13}$

In our study, there was a significant increase in image contrast with a decrease in VMI energy. The image contrast was maximum in keV VMI, followed in decreasing order from 50 to $70 \mathrm{keV}$ images, and a further decrease in image contrast was noted in the PEI. The mean tumor CNR value ranged from $2.525 \pm 1.7083$ in VMI at $40 \mathrm{keV}$ to $1.031 \pm 1.3967$ in PEI, approximately 1.5 times higher. The mean tumor CNR between VMI at $70 \mathrm{keV}(1.439 \pm 1.0859)$ and the PEI was also statistically significant. Subjective analysis revealed that 3.8 and $36.3 \%$ of the lesions were undetectable and suboptimal, respectively, in the PEI due to reduced image contrast. However, no lesions were undetectable due to reduced image contrast in the 40,50 , and $60 \mathrm{keV}$ images; one lesion was under the suboptimal category in 40 and $50 \mathrm{keV}$ images.

The statistically significant difference in tumor-to-liver contrast and tumor CNR can be explained by the difference in iodine concentrations between arterially hyper-enhancing focal liver lesions, and hepatic parenchyma at the late arterial phase emphasized at lower energies because of its proximity to the iodine K-edge. ${ }^{14} \mathrm{~A}$ gradual increase in image noise was noted in the VMI as it decreased from 70 to $40 \mathrm{keV}$. However, this increase in noise lost significance by the increased tumor-to-liver contrast in VMI, making CNR better in VMI.
This increased CNR helped to improve the lesion delineation and resulted in better detection performance in VMI ( - Figs. 3 and $\mathbf{4}$ ).

Different investigators have demonstrated a progressive increase of liver parenchyma and hypervascular liver lesion CNRs from higher to lower energy levels in monoenergetic images, reaching the peak at $40 \mathrm{keV} .^{12,15}$ Marin et al demonstrated that the maximal tumor-to-liver CNR for hypervascular lesion occurred at $40 \mathrm{keV}$ in phantom and in vivo. ${ }^{16}$

Considering the diagnostic confidence for lesion detection, $10 \%$ of the lesions were undetectable and $26.3 \%$ of the lesions were suboptimal in PEI, followed by $23.8 \%$ suboptimal detection in $70 \mathrm{keV}$ VMI. However, the diagnostic confidence was average or above average for all lesions in 40 to $60 \mathrm{keV}$ images (-Fig. 5). A meta-analysis by Lee et al ${ }^{17}$ with SECT showed a summarized sensitivity for the hypervascular liver lesion detection of $82 \%$ for lesions $\geq 1 \mathrm{~cm}$ and only $31 \%$ for lesions $<1 \mathrm{~cm}$. In our study, 19 lesions were less than $1 \mathrm{~cm}$; all were detected on VMI at 40 and $50 \mathrm{keV}$ (-Fig. 6). However, all these lesions were sub-optimal/undetectable in PEI. Similarly, De Cecco et $\mathrm{al}^{13}$ also concluded that $50 \mathrm{keV} \mathrm{VMI}+$ provides superior image quality and diagnostic accuracy for detecting hypervascular liver lesions with a diameter $<1 \mathrm{~cm}$ compared with linearly blended reconstructions.

VMI's improved diagnostic performance is presumably related to the higher CNR, which allows for improved lesion detection. The overall detection performance of all VMI was significantly better than PEI $(p<0.01)$. These results concur with previously published studies that analyzed the standard VMI algorithm. Yu et al reported that $70 \mathrm{keV}$ traditional monoenergetic images showed higher detectability of HCCs 

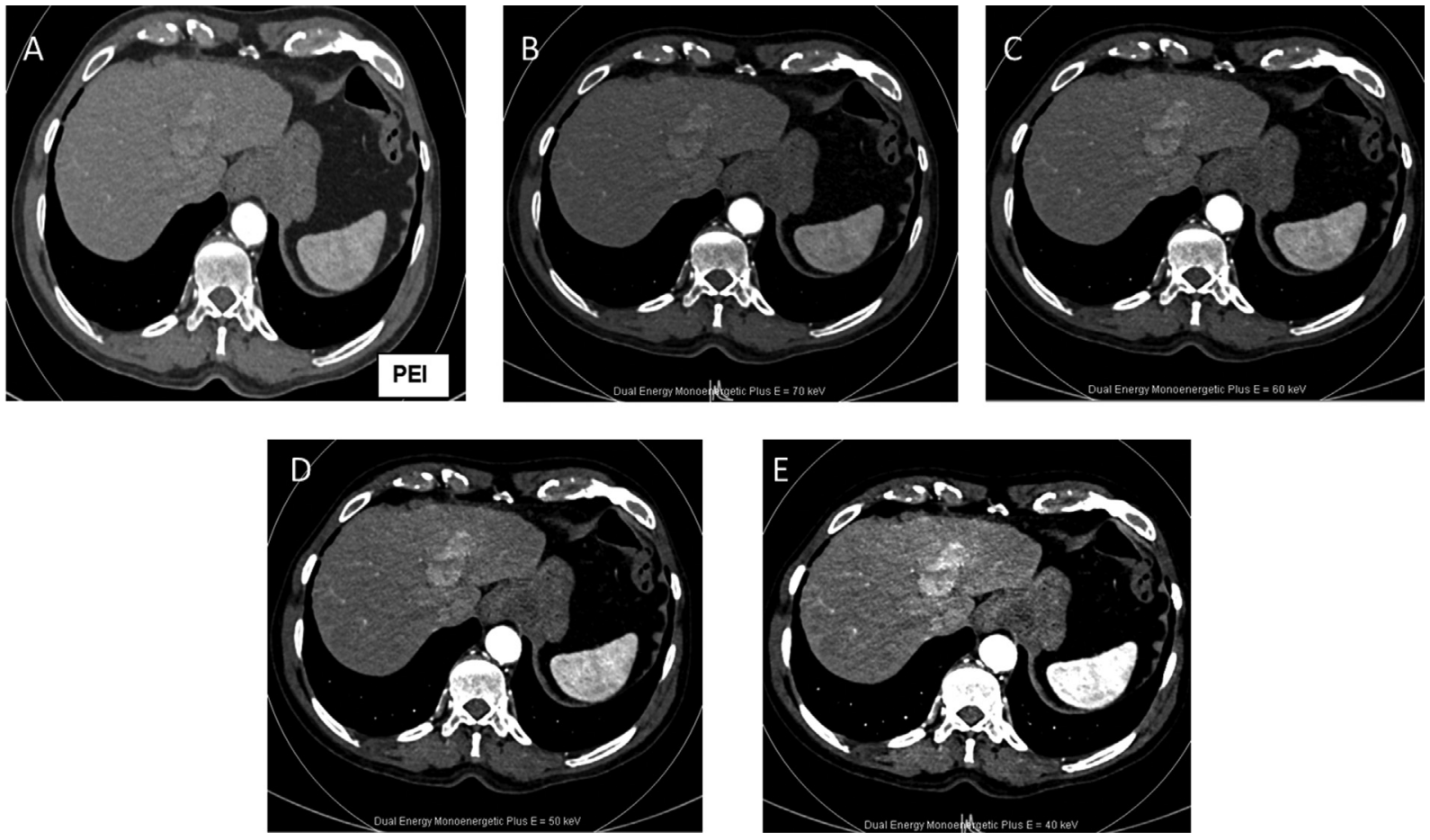

Fig. 4 Axial contrast-enhanced CT images of a patient with cirrhosis in the late arterial phase (A-PEI and B to E-VMI of 70-40 keV) show a LIRADS 5 lesion in segment IVa (proved as HCC). The lesion was suboptimally detected in the PEI; however, well seen in all the VMI and the conspicuity of lesion increases as VMI energy decreases. HCC, hepatocellular carcinoma; LIRADS, Liver Imaging Reporting and Data System; PEI, polychromatic energy image; VMI, virtual monoenergetic image.
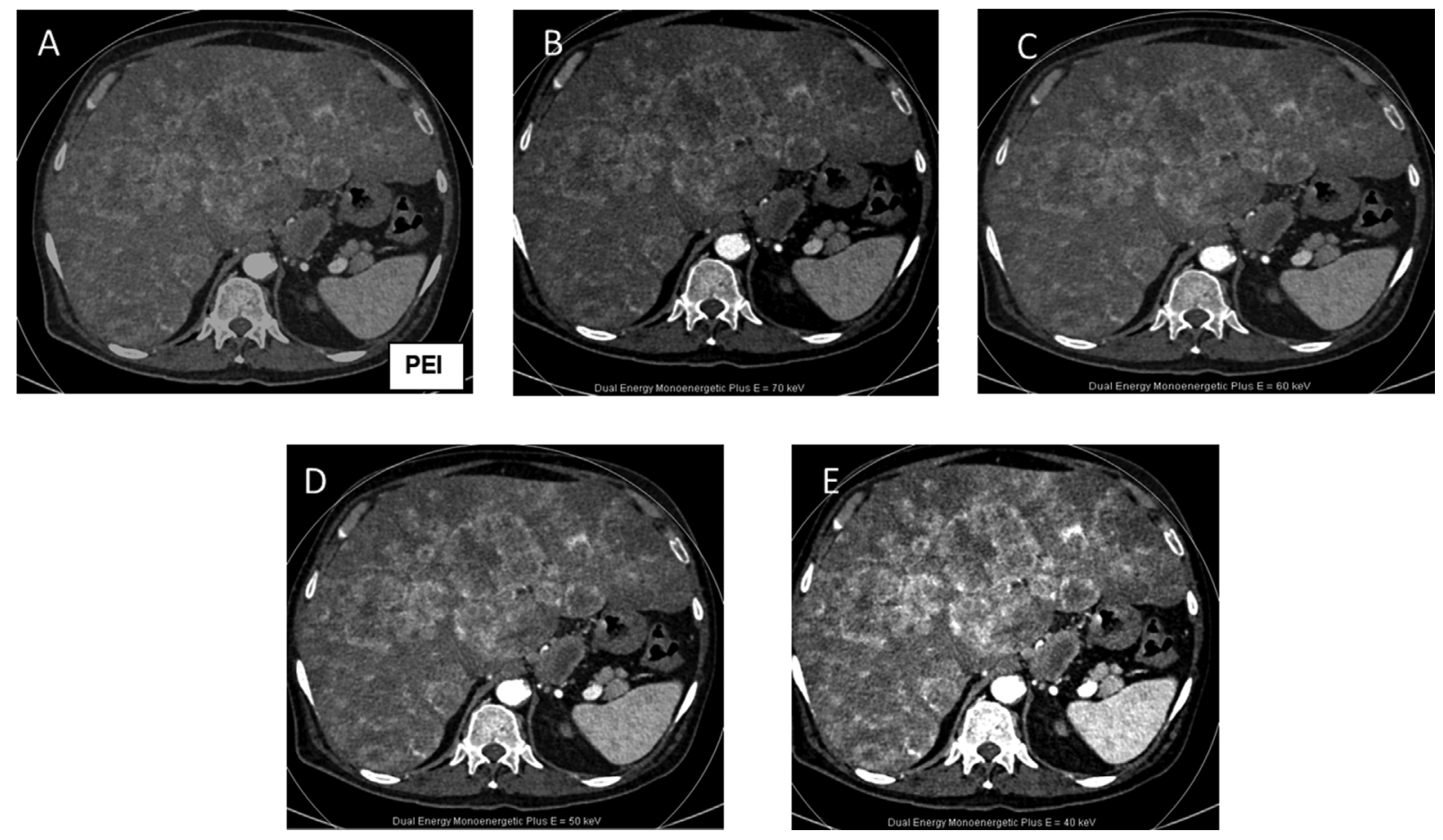

Fig. 5 Axial contrast-enhanced CT images of a patient with breast carcinoma in the arterial phase (A-PEl and B to E-VMI of 70-40 keV). The section of liver at the same level shows multiple lesions of varying size in both lobes of liver. Lesions were detectable in all sets of image; however, increased diagnostic confidence and extra small lesions were observed in the VMI (more in the $40 \mathrm{keV}$ image). PEI, polychromatic energy image; VMI, virtual monoenergetic image.

than conventional polyenergetic images. ${ }^{18}$ Similar results were reported by Lv et al who found that monoenergetic energy levels from 40 to $70 \mathrm{keV}$ increased the detectability of small HCC lesions compared with conventional $140 \mathrm{kVp}$ images, with the highest CNR found at $70 \mathrm{keV}^{2}$ A study done by Shuman et al showed that DECT images result in the greatest subjective lesion conspicuity with the radiation dose closer to that of SECT. ${ }^{4}$ 

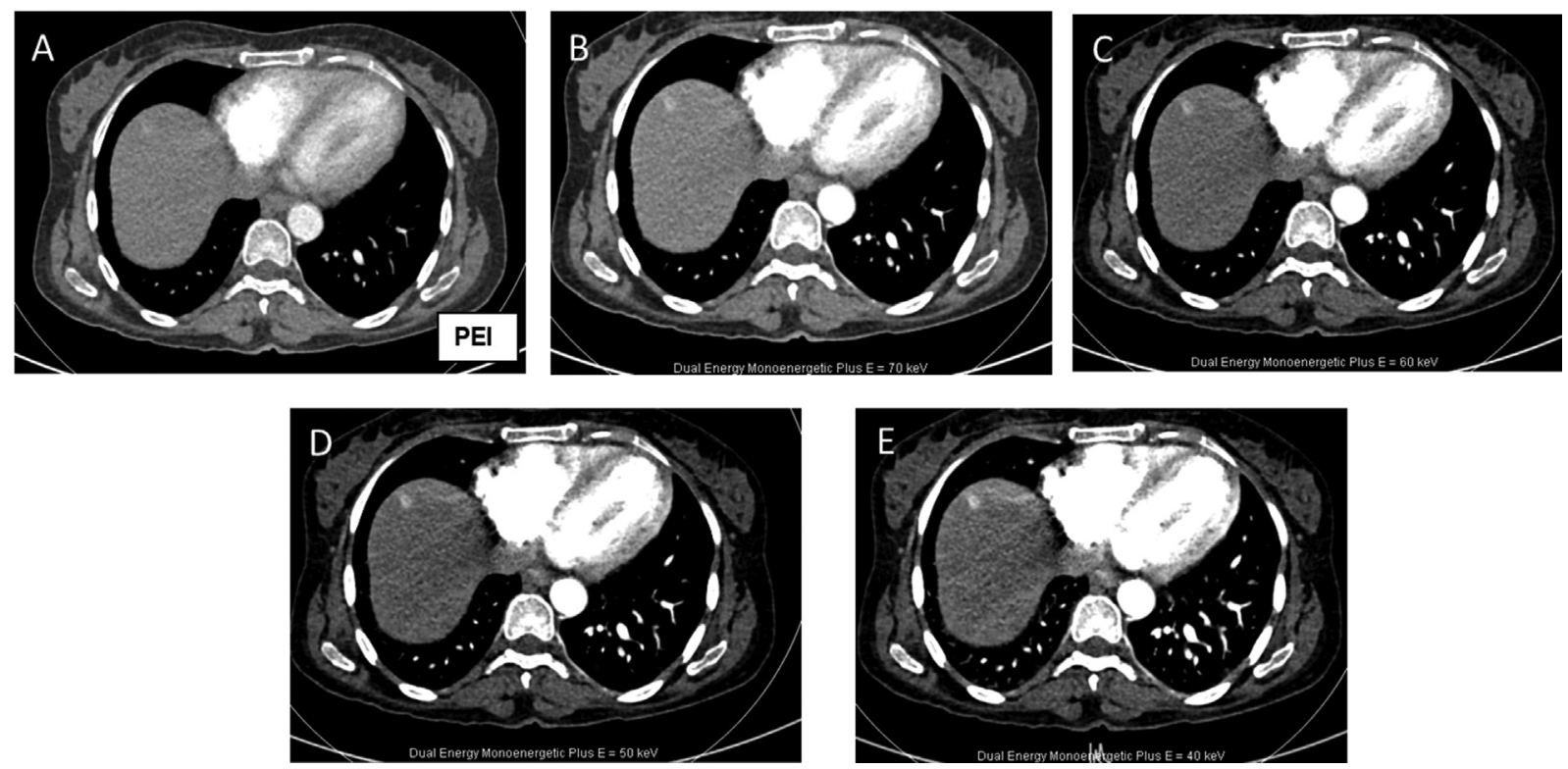

Fig. 6 Axial CT images in the arterial phase (A-PEI and B to E-VMI of 70-40 keV energy) in a patient with proven neuroendocrine tumor show a less than $1 \mathrm{~cm}$ lesion in the segment VIII of the liver. This lesion was not detectable (least diagnostic confidence) in the PEI; however, it was detected with good diagnostic confidence in the entire VMI. PEI, polychromatic energy image; VMI, virtual monoenergetic image.

This study had few limitations. The study population was relatively small. More extensive studies are necessary to confirm our results. Histological information was not available for all the detected lesions. However, this study was exclusively designed to evaluate monoenergetic imaging in detecting hypervascular malignant liver lesions, irrespective of histopathology. The results are currently only valid for DECT and post-processing techniques using dual-source $\mathrm{CT}$ technology and cannot automatically translate to DECT solutions by other vendors.

\section{Conclusion}

Our results indicate that a 40 to $70 \mathrm{keV}$ VMI dataset from a third-generation DSCT provides superior diagnostic accuracy for detecting hypervascular liver lesions despite the increased image noise. Considering that the image noise is more in the 40 and $50 \mathrm{keV}$ dataset and few of the lesions less than $1 \mathrm{~cm}$ were suboptimal in the $70 \mathrm{keV}$ dataset, it is better to evaluate the $60 \mathrm{keV}$ dataset for increased liver lesions detection with good image quality. However, it is also advisable to evaluate the $40 \mathrm{keV}$ images to detect small liver lesions ( $<1 \mathrm{~cm}$ size), which are suboptimal and undetectable in the higher energy VMI.

\section{Conflict of Interest}

None declared.

\section{References}

1 Altenbernd J, Heusner TA, Ringelstein A, Ladd SC, Forsting M, Antoch G. Dual-energy-CT of hypervascular liver lesions in patients with HCC: investigation of image quality and sensitivity. Eur Radiol 2011;21(04):738-743

2 Lv P, Lin XZ, Chen K, Gao J. Spectral CT in patients with small HCC: investigation of image quality and diagnostic accuracy. Eur Radiol 2012;22(10):2117-2124
3 Mileto A, Nelson RC, Samei E, et al. Dual-energy MDCT in hypervascular liver tumors: effect of body size on selection of the optimal monochromatic energy level. AJR Am J Roentgenol 2014; 203(06):1257-1264

4 Shuman WP, Green DE, Busey JM, et al. Dual-energy liver CT: effect of monochromatic imaging on lesion detection, conspicuity, and contrast-to-noise ratio of hypervascular lesions on late arterial phase. AJR Am J Roentgenol 2014;203(03):601-606

5 Große Hokamp N, Höink AJ, Doerner J, et al. Assessment of arterially hyper-enhancing liver lesions using virtual monoenergetic images from spectral detector $\mathrm{CT}$ : phantom and patient experience. Abdom Radiol (NY) 2018;43(08):2066-2074

6 Yeh BM, Shepherd JA, Wang ZJ, Teh HS, Hartman RP, Prevrhal S. Dual-energy and low-kVp CT in the abdomen. AJR Am J Roentgenol 2009;193(01):47-54

7 Yamada Y, Jinzaki M, Tanami Y, Abe T, Kuribayashi S. Virtual monochromatic spectral imaging for the evaluation of hypovascular hepatic metastases: the optimal monochromatic level with fast kilovoltage switching dual-energy computed tomography. Invest Radiol 2012;47(05):292-298

8 Sudarski S, Apfaltrer P, Nance JW Jr, et al. Objective and subjective image quality of liver parenchyma and hepatic metastases with virtual monoenergetic dual-source dual-energy CT reconstructions: an analysis in patients with gastrointestinal stromal tumor. Acad Radiol 2014;21(04):514-522

9 Caruso D, De Cecco CN, Schoepf UJ, et al. Can dual-energy computed tomography improve visualization of hypoenhancing liver lesions in portal venous phase? Assessment of advanced image-based virtual monoenergetic images. Clin Imaging 2017; 41:118-124

10 Husarik DB, Gordic S, Desbiolles L, et al. Advanced virtual monoenergetic computed tomography of hyperattenuating and hypoattenuating liver lesions: ex-vivo and patient experience in various body sizes. Invest Radiol 2015;50(10):695-702

11 Nagayama Y, Nakaura T, Oda S, et al. Dual-layer DECT for multiphasic hepatic CT with 50 percent iodine load: a matched-pair comparison with a $120 \mathrm{kVp}$ protocol. Eur Radiol 2018;28(04): $1719-1730$

12 Sellerer T, Noël PB, Patino M, et al. Dual-energy CT: a phantom comparison of different platforms for abdominal imaging. Eur Radiol 2018;28(07):2745-2755 
13 De Cecco CN, Caruso D, Schoepf UJ, et al. A noise-optimized virtual monoenergetic reconstruction algorithm improves the diagnostic accuracy of late hepatic arterial phase dual-energy CT for the detection of hypervascular liver lesions. Eur Radiol 2018;28(08): 3393-3404

14 Robinson E, Babb J, Chandarana H, Macari M. Dual source dual energy MDCT: comparison of $80 \mathrm{kVp}$ and weighted average $120 \mathrm{kVp}$ data for conspicuity of hypo-vascular liver metastases. Invest Radiol 2010;45(07):413-418

15 Kalisz K, Rassouli N, Dhanantwari A, Jordan D, Rajiah P. Noise characteristics of virtual monoenergetic images from a novel detector-based spectral CT scanner. Eur J Radiol 2018;98:118-125
16 Marin D, Ramirez-Giraldo JC, Gupta S, et al. Effect of a noiseoptimized second-generation monoenergetic algorithm on image noise and conspicuity of hypervascular liver tumors: an in vitro and in vivo study. AJR Am J Roentgenol 2016;206(06): 1222-1232

17 Leng S, Yu L, Fletcher JG, McCollough $\mathrm{CH}$. Maximizing iodine contrast-to-noise ratios in abdominal CT imaging through use of energy domain noise reduction and virtual monoenergetic dualenergy ct. Radiology 2015;276(02):562-570

$18 \mathrm{Yu} \mathrm{Y,} \mathrm{Lin} \mathrm{X,} \mathrm{Chen} \mathrm{K,} \mathrm{et} \mathrm{al.} \mathrm{Hepatocellular} \mathrm{carcinoma} \mathrm{and} \mathrm{focal}$ nodular hyperplasia of the liver: differentiation with CT spectral imaging. Eur Radiol 2013;23(06):1660-1668 